\title{
List of referees
}

(C) Japanese Society of Nephrology 2013

List of reference who provided reviews for CEN Case Reports during the 2012-2013 period.

\begin{tabular}{|c|c|c|c|c|}
\hline Abe, Hideharu & (1) & Igarashi, Takashi & (1) & Koike, Junki \\
\hline Abe, Michiaki & (2) & Igarashi, Tatsuo & (1) & Komatsu, Yasuhiro \\
\hline Aikawa, Atsushi & (2) & Iijima, Kazumoto & (6) & Komatsuda, Atsushi \\
\hline Akai, Yasuhiro & (4) & Ikegaya, Naoki & (4) & Kondo, Shuij \\
\hline Ando, Ryoichi & (3) & Ikezumi, Yohei & (5) & Kondo, Tsunenori \\
\hline Arimura, Yoshihiro & (2) & Imai, Hirokazu & (6) & Konta, Tsuneo \\
\hline Awazu, Midori & (3) & Imasawa, Toshiyuki & (1) & Koshikawa, Masao \\
\hline Aya, Kunihiko & (4) & Inaguma, Daijo & (3) & Koya, Daisuke \\
\hline Ehara, Takashi & (4) & Iseki, Kunitoshi & (1) & Kumagai, Hiromichi \\
\hline Endoh, Masayuki & (2) & Ishibashi, Kenichi & (3) & Kuriyama, Satoru \\
\hline Fujigaki, Yoshihide & (2) & Ishibashi, Michio & (4) & Kusano, Eiji \\
\hline Fujii, Yasuhisa & (1) & Ishikawa, San-e & (4) & Lerma, Edgar, V \\
\hline Fujimoto, Shouichi & (7) & Ishikura, Kenji & (2) & Matsui, Satoshi \\
\hline Fujimura, Yoshihiro & (3) & Ishizu, Akihiro & (2) & Minakami, Hisanori \\
\hline Fujisawa, Masato & (2) & Itabashi, Mitsuyo & (4) & Miura, Kenichiro \\
\hline Fujita, Takayuki & (3) & Ito, Takafumi & (5) & Miura, Kenichirou \\
\hline Fukatsu, Atsushi & (2) & Ito, Takahito & (1) & Miura, Naoto \\
\hline Fukazawa, Yuichiro & (1) & Ito, Yasuhiko & (2) & Miyazaki, Masanobu \\
\hline Fukuuchi, Fumiko & (1) & Iwano, Masayuki & (4) & Mochizuki, Toshio \\
\hline Goto, Shin & (3) & Joh, Kensuke & (1) & Monkawa, Toshiaki \\
\hline Hanaoka, Kazushige & (7) & Kaito, Hiroshi & (9) & Mori, Kiyoshi \\
\hline Hara, Taiga & (1) & Kamata, Kouju & (2) & Mori, Yasukiyo \\
\hline Harabuchi, Yasuaki & (2) & Kaname, Shinya & (18) & Morimoto, Satoshi \\
\hline Hasegawa, Hajime & (5) & Kaneko, Kazunari & (3) & Morozumi, Kunio \\
\hline Hashizume, Hiroshi & (1) & Kanno, Yoshihiko & (5) & Mugiya, Soichi \\
\hline Hayashi, Matsuhiko & (4) & Kashihara, Naoki & (2) & Mukoyama, Masashi \\
\hline Hayashi, Terumasa & (1) & Katafuchi, Ritsuko & (6) & Murata, Tomohiro \\
\hline Higuchi, Makoto & (7) & Kato, Akihiko & (5) & Muso, Eri \\
\hline Hiki, Yoshiyuki & (3) & Kato, Hitosi & (3) & Muto, Satoru \\
\hline Hirahashi, Junichi & (2) & Kawanishi, Hideki & (2) & Nagasawa, Yasuyuki \\
\hline Hiramatsu, Makoto & (1) & Kawano, Mitsuhiro & (1) & Nagata, Michio \\
\hline Hirawa, Nobuhito & (8) & Kawasaki, Yukihiko & (4) & Nakajima, Mitsuru \\
\hline Hirayama, Kouichi & (19) & Kazama, Junichiro, James & (2) & Nakamoto, Hidetomo \\
\hline Hiromura, Keiju & (3) & Kitamura, Hiroshi & (5) & Nakamura, Satoko \\
\hline Hisano, Satoshi & (1) & Kitamura, Kenichiro & (1) & Nakamura, Tsukasa \\
\hline Homma, Sakae & (2) & Kobayashi, Masaki & (5) & Nakanishi, Koichi \\
\hline Horikoshi, Satoshi & (1) & Kobayashi, Shuzo & (4) & Nakayama, Masaaki \\
\hline
\end{tabular}


Nangaku, Masaomi

Narita, Ichiei

Negi, Shigeo

Niimura, Fumio

Nishi, Shinichi

Nishikawa, Megumi

Nishio, Saori

Nitta, Kosaku

Noiri, Eisei

Nomura, Shinsuke

Nonoguchi, Hiroshi

Nozu, Kandai

Nutahara, Kikuo

Oda, Takashi

Ogata, Hiroaki

Ogawa, Yoshihide

Ogura, Makoto

Ohashi, Kenichi

Ohno, Iwao

Ohtomo, Yoshiyuki

Okada, Hirokazu

Okuda, Seiya

Ono, Takahiko

Osawa, Hiroshi

Sada, Ken-ei

Saeki, Takako

Saito, Kazuhide

Saito, Takao
(2) Sakai, Ken

(1) Samma, Shoji

(5) Sasaki, Satoshi

(5) Sasaki, Tamaki

(6) Sato, Hiroshi

(1) Sekine, Takashi

(1) Shibagaki, Yugo

(4) Shigematsu, Takashi

(1) Shikata, Kenichi

(4) Shimazui, Toru

(2) Shimizu, Akira

(4) Shinoda, Toshio

(1) Soma, Jun

(1) Sugimoto, Toshiro

(2) Taguchi, Takashi

(1) Takashi, Oda

(1) Takayama, Tatsuya

(2) Takeda, Tetsuro

(1) Takemura, Tsukasa

(1) Takenaka, Tsuneo

(2) Takeoka, Hiroya

(2) Tanaka, Hiroshi

(2) Tanaka, Mari

(1) Tanaka, Motoko

(1) Terawaki, Hiroyuki

(4) Tervaert, Jan. W. Cohen

(3) Tojo, Akihiro

(2) Tomita, Shigeki
(4) Tomo, Tadashi

(1) Tsuchiya, Ken

(1) Tsukahara, Hirokazu

(5) Tsukamoto, Yusuke

(13) Tsuruya, Kazuhiko (1)

(5) Ubara, Yoshifumi

(5) Uchida, Shinichi

(1) Uda, Susumu

(1) Uesugi, Noriko (5)

(1) Uozumi, Jiro

(6) Usui, Joichi

(2) Uzu, Takashi

(6) Wakasugi, Minako (2)

(3) Wakui, Hideki

(1) Yamabe, Hideaki

(2) Yamada, Akira

(1) Yamamoto, Tatsuo

(1) Yamanaka, Nobuaki

(2) Yasuda, Hideo

(3) Yasuda, Takashi

(2) Yokoyama, Hitoshi

(1) Yorioka, Noriaki

(1) Yoshida, Atsuhiro

(3) Yoshida, Masaharu

(6) Yumura, Wako

(1) Yuzawa, Yukio

(2) (), number of reviewed articles. 